\title{
A stability result via Carleman estimates for an inverse source problem related to a hyperbolic integro-differential equation
}

\author{
CECILIA CAVATERRA ${ }^{1}$, ALFREDO LORENZI ${ }^{2}$ \\ and MASAHIRO YAMAMOTO ${ }^{3}$ \\ 1,2 Dipartimento di Matematica "F. Enriques”, Università degli Studi di Milano \\ Via Saldini 50, 20133 Milano, Italy \\ ${ }^{3}$ Department of Mathematical Sciences, The University of Tokyo \\ 3-8-1 Komaba, Meguro, Tokyo 153 Japan \\ E-mails: cecilia.cavaterra@mat.unimi.it / alfredo.lorenzi@mat.unimi.it / \\ myama@ms.u-tokyo.ac.jp
}

\begin{abstract}
First we prove a Carleman estimate for a hyperbolic integro-differential equation. Next we apply such a result to identify a spatially dependent function in a source term by an (additional) single measurement on the boundary.
\end{abstract}

Mathematical subject classification: 45Q05, 45K05.

Key words: Hyperbolic integro-differential equation, Carleman estimate, inverse source problem, stability estimate.

\section{Introduction and main results}

Let $\Omega \subset \mathbb{R}^{n}$ be a bounded domain with smooth boundary $\partial \Omega$ and let $v=v(x)$ be the outward unit normal vector to $\partial \Omega$ at $x, \partial_{\nu} u=\nabla u \cdot v$. We consider a hyperbolic integro-differential equation:

$$
\begin{gathered}
(P u)(x, t) \equiv \partial_{t}^{2} u(x, t)-p(x) \Delta u(x, t)-\int_{0}^{t} K(x, t, \eta) \Delta u(x, \eta) d \eta \\
-L(u)(x, t)=F(x, t), \quad x \in \Omega, t>0,
\end{gathered}
$$


where

$$
\begin{aligned}
L(u)(x, t)= & \sum_{j=1}^{n} q_{j}(x) \partial_{j} u(x, t)+q_{n+1}(x) \partial_{t} u(x, t)+q_{0}(x) u(x, t) \\
& +\sum_{j=1}^{n} \int_{0}^{t} H_{j}(x, t, \eta) \partial_{j} u(x, \eta) d \eta \\
& +\int_{0}^{t} H_{n+1}(x, t, \eta) \partial_{t} u(x, \eta) d \eta \\
& +\int_{0}^{t} H_{0}(x, t, \eta) u(x, \eta) d \eta .
\end{aligned}
$$

Here $p \in C^{2}(\bar{\Omega}), p>0$ on $\bar{\Omega}, q_{j} \in C(\bar{\Omega}), j=0, \ldots, n+1, K \in C^{2}(\bar{\Omega} \times E(T))$, $H_{j} \in C(\bar{\Omega} \times E(T)), j=0, \ldots, n+1$ such that $\partial_{t} H_{j} \in C(\bar{\Omega} \times E(T))$. Here we set $E(T)=\left\{(t, \eta) \in \mathbb{R}^{2}: 0 \leq \eta \leq t \leq T\right\}$.

We set

$$
\begin{gathered}
x=\left(x_{1}, \ldots, x_{n}\right), \partial_{t}=\frac{\partial}{\partial t}, \partial_{j}=\frac{\partial}{\partial x_{j}}, j=1,2, \ldots, n, \\
\nabla_{x, t}=\left(\nabla, \partial_{t}\right)=\left(\partial_{1}, \ldots, \partial_{n}, \partial_{t}\right), \Delta=\sum_{j=1}^{n} \partial_{j}^{2} .
\end{gathered}
$$

Equation (1.1) appears in various cases such as viscoelasticity.

One of the fundamental questions for (1.1) is the unique continuation: if $u$ satisfies (1.1) and $u=\partial_{\nu} u=0$ on $\Gamma \times(0, T)$ where $\Gamma \subset \partial \Omega$, then can we choose a neighbourhood $U \subset \mathbb{R}^{n}$ of $\Gamma$ and an interval $I \subset(0, T)$ such that $u=0$ in $U \times I$ ?

In order to prove the unique continuation and discuss applications to inverse problems, a Carleman estimate is a main tool. In this paper, we will establish a Carleman estimate for (1.1), and will apply it to determine an unknown source term. We stress that our result is the first step to determine $x$-dependent coefficients in (1.1). In a forthcoming paper we will discuss more general inverse problems.

In addition to the assumption that $p \in C^{2}(\bar{\Omega})$ and $p(x)>0$ in $\bar{\Omega}$, throughout this paper we suppose that there exists $x_{0} \in \mathbb{R}^{n} \backslash \bar{\Omega}$ such that

$$
\frac{1}{2} p(x)^{2}-\left(\nabla p(x) \cdot\left(x-x_{0}\right)\right) \geq 0, \quad x \in \bar{\Omega} \text {. }
$$


We set

$$
\varphi(x, t)=\left|x-x_{0}\right|^{2}-\beta t^{2},
$$

where $\beta>0$ is a sufficiently small constant depending on $\Omega, p, x_{0}$. Furthermore, for a fixed $R>0$ and any $\varepsilon>0$, let

$$
\begin{aligned}
& Q(\varepsilon)=\left\{(x, t) \in \Omega \times(0, \infty): \varphi(x, t)>R^{2}+\varepsilon\right\}, \\
& \Omega(\varepsilon)=\left\{x \in \Omega:\left|x-x_{0}\right|>\left(R^{2}+\varepsilon\right)^{1 / 2}\right\} .
\end{aligned}
$$

Then we can show

Theorem 1 (Carleman estimate). Let $u \in H^{2}(Q(\varepsilon))$ satisfy (1.1) and

$$
u(x, 0)=0 \quad \text { or } \quad \partial_{t} u(x, 0)=K(x, 0,0)=0, \quad x \in \Omega(0)
$$

Then there exist $s_{0}>0$ and a constant $C=C\left(s_{0}\right)>0$ independent of $u$ such that

$$
\begin{gathered}
\int_{Q(\varepsilon)}\left(s\left|\nabla_{x, t} u\right|^{2}+s^{3} u^{2}\right) e^{2 s \varphi} d x d t \\
\leq C \int_{Q(\varepsilon)}|F|^{2} e^{2 s \varphi} d x d t+C e^{C s}\|u\|_{(1), \Sigma}^{2}
\end{gathered}
$$

for any $s \geq s_{0}$, where $\Sigma=\partial Q(\varepsilon) \backslash(\Omega(\varepsilon) \times\{0\})$ and

$$
\|u\|_{(1), \Sigma}^{2}=\int_{\partial Q(\varepsilon) \backslash(\Omega(\varepsilon) \times\{0\})}\left(\left|\nabla_{x, t} u\right|^{2}+u^{2}\right) d S .
$$

Remark 1. Condition $K(x, 0,0)=0$ in (1.5) can be erased if we are given the initial conditions $u(x, 0)=\partial_{t} u(x, 0)=0, x \in \Omega(0)$.

Remark 2. In the weight function $\varphi$, we have to choose $\beta=\beta\left(\Omega, p, x_{0}\right)>0$ sufficiently small. In particular, if $p \equiv 1$, then we can choose any $\beta \in(0,1)$ (e.g., [14], [20]).

Inequality (1.6) is called a Carleman estimate. Carleman estimates are wellknown for elliptic, parabolic and hyperbolic operators (e.g., Hörmander [8], 
Isakov [12]-[14], Klibanov and Timonov [20], Lavrent'ev, Romanov and Shishat'skiǔ [23]). However our system is involved with the integral term

$$
\int_{0}^{t} K(x, t, \eta) \Delta u(x, \eta) d \eta
$$

so that a Carleman estimate for (1.1) is not found in the existing papers. In Yong and Zhang [31], an exact controllability problem is considered for a related system.

In order to treat the integral term (1.7), we have to assume the extra information (1.5). In other words, a usual Carleman estimate is proved for the extended domain

$$
\left\{(x, t) \in \Omega \times[-T, T]: \varphi(x, t)>R^{2}+\varepsilon\right\},
$$

but not for

$$
\left\{(x, t) \in \Omega \times[0, T]: \varphi(x, t)>R^{2}+\varepsilon\right\} .
$$

In order to apply a usual Carleman estimate to the inverse problem in $t>0$, we should extend the solution $u$ to $t<0$. Such an extension requires an extra argument owing to (1.7). On the contrary, for an inverse problem over a time interval $(0, T)$ under $(1.5)$, we need not extend $u$ to $(-T, 0)$, and can directly apply our Carleman estimate (1.6). This kind of Carleman estimates in $t>0$ is derived by a pointwise inequality in Klibanov and Timonov [20], Lavrent'ev, Romanov and Shishat'skiı̌ [23], and is quite different from the Carleman estimates in Hörmander [8], Isakov [12]-[14], etc.

Next we will consider

The Inverse Source Problem. Let $\varepsilon>0$ be arbitrarily fixed and let $r \in$ $W^{1, \infty}\left(0, T ; L^{\infty}(\Omega)\right)$ be a given function. Let us consider

$$
\begin{gathered}
(P u)(x, t)=r(x, t) f(x), \quad x \in \Omega, \quad 0<t<T, \\
u(x, 0)=\partial_{t} u(x, 0)=0, \quad x \in \Omega .
\end{gathered}
$$

Our task is to determine function $f \in \Omega(\delta)$ with $\delta>0$ from the knowledge of

$$
\left.u\right|_{\Gamma \times(0, T)},\left.\quad \partial_{\nu} u\right|_{\Gamma \times(0, T)} .
$$

Here $\Gamma$ is an open subset of $\partial \Omega$. 
The problem to be solved is actually a sort of "double Cauchy" problem, since we are given Cauchy conditions on both $t=0$ and $\Gamma$. Note that we are given only "incomplete" boundary conditions, since no conditions on $u$ and its derivatives are prescribed on the whole of $\partial \Omega$.

Let us assume

$$
\overline{\Omega(0)} \subset \Omega \cup \bar{\Gamma} .
$$

We are ready to state the stability result for our inverse source problem.

Theorem 2. Let $u \in C^{3}\left([0, T] ; L^{2}(\Omega)\right) \cap C^{2}\left([0, T] ; H^{1}(\Omega)\right) \cap C^{1}([0, T]$; $H^{2}(\Omega)$ ) satisfy (1.8) and (1.9), and let us assume in addition to the regularity assumptions for the coefficients in (1.1) that $\partial_{t} K \in C^{2}(\bar{\Omega} \times E(T))$. We further assume

$$
|r(x, 0)|>0, \quad \text { for all } x \in \bar{\Omega}
$$

and

$$
T>\frac{\sup _{x \in \Omega(0)}\left|x-x_{0}\right|}{\sqrt{\beta}} .
$$

Then for any $\delta>0$, there exist two constants $C=C\left(\Omega, T, p, x_{0}, \beta, \delta, r, R\right)>0$ and $\kappa=\kappa\left(\Omega, T, p, x_{0}, \beta, \delta, r, R\right) \in(0,1), \beta$ and $R$ being as in (1.3) and (1.4), such that

$$
\begin{aligned}
\|f\|_{L^{2}(\Omega(\delta))} \leq & C\left(\|u\|_{H^{1}(Q(0))}+\left\|\partial_{t} u\right\|_{H^{1}(Q(0))}+\|f\|_{L^{2}(\Omega(0))}\right)^{1-\kappa} \\
& \times\left(\|u\|_{H^{1}(\Gamma \times(0, T))}+\left\|\partial_{t} u\right\|_{H^{1}(\Gamma \times(0, T))}\right)^{\kappa} \\
& +C\left(\|u\|_{H^{1}(\Gamma \times(0, T))}+\left\|\partial_{t} u\right\|_{H^{1}(\Gamma \times(0, T))}\right) .
\end{aligned}
$$

The factor $\left(\|u\|_{H^{1}(\Gamma \times(0, T))}+\left\|\partial_{t} u\right\|_{H^{1}(\Gamma \times(0, T))}\right)$ is the observation datum and (1.13) shows the stability of Hölder type which is conditional under an a priori boundedness of $\left(\|u\|_{H^{1}(Q(0))}+\left\|\partial_{t} u\right\|_{H^{1}(Q(0))}+\|f\|_{L^{2}(\Omega(0))}\right)$.

Theorem 2 is derived from Theorem 1 by means of the method created by Bukhgeim and Klibanov [3].

As related works on inverse problems by Carleman estimates, see Bellassoued [1], Bukhgeim [2], Imanuvilov and Yamamoto [9]-[11], Isakov [12][14], Khaĭdarov [18], Klibanov [19], Klibanov and Timonov [20], Klibanov and Yamamoto [21], Kubo [22], Yamamoto [30] and the references therein.

The novelty of this paper in comparison with the quoted ones, consists in: 
(1) establishing a Carleman estimate for (1.1) with the integral term (Theorem 1).

(2) deriving a Hölder estimate for an unknown factor depending on $x$ in the source term of (1.8).

In particular, we can prove the Lipschitz stability for the unknown function $f$ in terms of the data measured on a suitably large part $\Gamma$ of $\partial \Omega$. The related proof follows some ideas contained in [9] and [10], and makes use of our Carleman estimate (Theorem 1). We stress that Theorem 1 is the starting point for establishing stability also for different inverse problems related to hyperbolic integro-differential equations, such as the determination of $p(x)$ in (1.1), which is physically important. For example, let $v=v(x, t)$ and $w=w(x, t)$ be the solutions to (1.1) corresponding respectively to the coefficients $p$ and $q$. Setting $u=v-w$, we obtain (1.1) where $F(x, t)$ is replaced by $(p(x)-q(x)) \Delta w(x, t)$. Then, on the basis of Theorem 1, we can apply an argument similar to the one used in [11] to prove the stability concerning $p(x)$. In a forthcoming paper, we discuss the details.

Different kinds of inverse problems, which consist in determining time-dependent factors in the kernel $K(x, t, \eta)$, are dealt with, e.g., in the papers by Cavaterra [4], Cavaterra and Grasselli [5], Cavaterra and Lorenzi [6], Janno and Lorenzi [15], Janno and von Wolfersdorf [16], Kabanikhin and Lorenzi [17], Lorenzi [24], Lorenzi and Messina [25], [26], Lorenzi and Romanov [27], Lorenzi and Yahkno [28], von Wolfersdorf [29] and the references therein.

The rest of this paper is composed of two sections: in Section 2 we will prove Theorem 1, while Section 3 is devoted to the proof of Theorem 2.

\section{Proof of Theorem 1}

Henceforth $C>0$ denotes generic constants which are independent of $s>0$ and may vary from line to line. We first state a pointwise Carleman estimate for a hyperbolic operator (Theorem 2.2.4 in Klibanov and Timonov [20, pp. 45-46]). See also Lemma 2 in [23, p. 128] for the case of $p \equiv 1$ and Cheng, Isakov, Yamamoto and Zhou [7]. 
Theorem A. Let $p=p(x) \in C^{2}(\bar{\Omega})$ satisfy (1.2) and let $\beta>0$ be sufficiently small. Then there exist constants $s_{0}>0$ and $C>0$ such that

$$
\begin{gathered}
\left(s\left|\nabla_{x, t} w(x, t)\right|^{2}+s^{3}|w(x, t)|^{2}\right) e^{2 s \varphi(x, t)}+\operatorname{div} U(x, t)+\partial_{t} V(x, t) \\
\leq C\left|\left(\partial_{t}^{2}-p(x) \Delta\right) w(x, t)\right|^{2} e^{2 s \varphi(x, t)}, \quad(x, t) \in Q(\varepsilon)
\end{gathered}
$$

for all $s \geq s_{0}$ and $w \in C^{2}(\overline{Q(\varepsilon)})$. Here $(U, V)$ is a vector-valued function and satisfies

$$
\begin{gathered}
|U(x, t)|+|V(x, t)| \\
\leq C e^{2 s \varphi(x, t)}\left(s\left|\nabla_{x, t} w(x, t)\right|^{2}+s^{3}|w(x, t)|^{2}\right), \quad(x, t) \in Q(\varepsilon) .
\end{gathered}
$$

Moreover $V(x, 0)=0, x \in \Omega(0)$ if $w(x, 0)=0$ or $\partial_{t} w(x, 0)=0, x \in \Omega(0)$.

Here we modify the statement of Theorem 2.2.4 in [20], the proof being essentially the same. Integrating the first inequality in the above theorem over $Q(\varepsilon)$ and making use of the properties of functions $U$ and $V$ in the proof of the same theorem, we obtain

Theorem B. Let $p=p(x) \in C^{2}(\bar{\Omega})$ satisfy (1.2), $\beta>0$ be sufficiently small and $w(x, 0)=0$ or $\partial_{t} w(x, 0)=0, x \in \Omega(0)$. Then there exist constants $s_{0}>0$ and $C>0$ such that

$$
\begin{aligned}
& \int_{Q(\varepsilon)}\left(s\left|\nabla_{x, t} w\right|^{2}+s^{3}|w|^{2}\right) e^{2 s \varphi} d x d t \\
& \leq C \int_{Q(\varepsilon)}\left|\left(\partial_{t}^{2}-p(x) \Delta\right) w\right|^{2} e^{2 s \varphi} d x d t \\
& \quad+C e^{C s} \int_{\partial Q(\varepsilon) \backslash(Q(\varepsilon) \cap\{t=0\})}\left(\left|\nabla_{x, t} w\right|^{2}+|w|^{2}\right) d S
\end{aligned}
$$

for any $s \geq s_{0}$ and any $w(x, t) \in C^{2}(\overline{Q(\varepsilon)})$.

Set

$$
v(x, t)=p(x) u(x, t)+\int_{0}^{t} K(x, t, \eta) u(x, \eta) d \eta, \quad x \in \Omega, t>0 .
$$


Then from the formulae

$$
\begin{aligned}
\partial_{t}^{2} v(x, t)= & p(x) \partial_{t}^{2} u(x, t)+\left\{\partial_{t}(K(x, t, t))+\partial_{t} K(x, t, t)\right\} u(x, t) \\
& +K(x, t, t) \partial_{t} u(x, t)+\int_{0}^{t} \partial_{t}^{2} K(x, t, \eta) u(x, \eta) d \eta, \\
\Delta v(x, t)= & p(x) \Delta u(x, t)+\int_{0}^{t} K(x, t, \eta) \Delta u(x, \eta) d \eta \\
& +2 \nabla p(x) \cdot \nabla u(x, t)+u(x, t) \Delta p(x) \\
& +2 \int_{0}^{t} \nabla K(x, t, \eta) \cdot \nabla u(x, \eta) d \eta+\int_{0}^{t} u(x, \eta) \Delta K(x, t, \eta) d \eta,
\end{aligned}
$$

we easily deduce that $v$ solves the equation

$$
\begin{aligned}
\partial_{t}^{2} v(x, t)-p(x) \Delta v(x, t)=p(x) F(x, t)+p(x) L(u)(x, t) \\
+\left\{\partial_{t}(K(x, t, t))+\partial_{t} K(x, t, t)-p(x) \Delta p(x)\right\} u(x, t) \\
+K(x, t, t) \partial_{t} u(x, t)-2 p(x) \nabla p(x) \cdot \nabla u(x, t) \\
+\int_{0}^{t}\left[\partial_{t}^{2} K(x, t, \eta)-p(x) \Delta K(x, t, \eta)\right] u(x, \eta) d \eta \\
\quad-2 p(x) \int_{0}^{t} \nabla K(x, t, \eta) \cdot \nabla u(x, \eta) d \eta \\
\equiv p(x) F(x, t)+L_{1}(u)(x, t), \quad x \in \Omega, t>0,
\end{aligned}
$$

and the initial conditions

$$
v(x, 0)=0 \quad \text { or } \quad \partial_{t} v(x, 0)=0, \quad x \in \Omega(0) .
$$

Here we note that $\left(\partial_{t} K\right)(x, t, t)=\left.\partial_{t} K(x, t, \eta)\right|_{\eta=t}$.

In terms of (2.3), we apply Theorem B to (2.2). Consequently there exists some positive constant $s \geq s_{0}$ such that, for $s \geq s_{0}$, we obtain

$$
\begin{aligned}
\int_{Q(\varepsilon)} & \left(s\left|\nabla_{x, t} v\right|^{2}+s^{3} v^{2}\right) e^{2 s \varphi} d x d t \\
\leq & C \int_{Q(\varepsilon)}|p F|^{2} e^{2 s \varphi} d x d t \\
& +C \int_{Q(\varepsilon)}\left|L_{1}(u)\right|^{2} e^{2 s \varphi} d x d t+C e^{C s}\|u\|_{(1), \Sigma}^{2}
\end{aligned}
$$


where $\Sigma=\partial Q(\varepsilon) \backslash(\Omega(\varepsilon) \times\{0\})$.

By our assumptions on the coefficients and the kernels we deduce the estimate

$$
\begin{aligned}
\left|L_{1}(u)(x, t)\right| \leq & C\left(\left|\nabla_{x, t} u(x, t)\right|+|u(x, t)|\right) \\
& +C \int_{0}^{t}\left(\left|\nabla_{x, t} u(x, \eta)\right|+|u(x, \eta)|\right) d \eta .
\end{aligned}
$$

Consequently, from (2.4) we obtain, for $s \geq s_{0}$,

$$
\begin{aligned}
\int_{Q(\varepsilon)} & \left(s\left|\nabla_{x, t} v\right|^{2}+s^{3} v^{2}\right) e^{2 s \varphi} d x d t \\
\leq & C \int_{Q(\varepsilon)}|F|^{2} e^{2 s \varphi} d x d t+C \int_{Q(\varepsilon)}\left(\left|\nabla_{x, t} u\right|^{2}+u^{2}\right) e^{2 s \varphi} d x d t \\
& +C \int_{Q(\varepsilon)}\left(\int_{0}^{t}\left(\left|\nabla_{x, t} u(x, \eta)\right|+|u(x, \eta)|\right) d \eta\right)^{2} e^{2 s \varphi} d x d t \\
& +C e^{C s}\|u\|_{(1), \Sigma}^{2} .
\end{aligned}
$$

We need now to show

\section{Lemma 1.}

$$
\int_{Q(\varepsilon)}\left(\int_{0}^{t}|w(x, \xi)| d \xi\right)^{2} e^{2 s \varphi} d x d t \leq \frac{C}{s} \int_{Q(\varepsilon)}|w(x, t)|^{2} e^{2 s \varphi} d x d t
$$

for all $w \in L^{2}(Q(\varepsilon))$.

Lemma 1 is fundamental in order to derive a Carleman estimate for our inverse problem. We note that it was proved in Bukhgeim and Klibanov [3], Klibanov [19], but with a factor not containing $1 / s$. On the contrary, for our proof the factor $1 / \mathrm{s}$ is essential. As for the proof of Lemma 1, see Lemma 3.1.1 (pp.77-78) in [20]. However, for completeness, we will give the proof of it in Appendix.

By (2.1) and $p>0$ on $\bar{\Omega}$, we obtain

$$
u(x, t)=\frac{1}{p(x)} v(x, t)-\int_{0}^{t} \frac{K(x, t, \eta)}{p(x)} u(x, \eta) d \eta .
$$

Hence, owing to Lemma 1, we have

$$
\int_{Q(\varepsilon)} u^{2} e^{2 s \varphi} d x d t \leq C \int_{Q(\varepsilon)} v^{2} e^{2 s \varphi} d x d t+\frac{C}{s} \int_{Q(\varepsilon)} u^{2} e^{2 s \varphi} d x d t .
$$


Taking $s>s_{0}$ sufficiently large, we can absorb the second term on the right hand side into the left hand side, and we have

$$
\int_{Q(\varepsilon)} u^{2} e^{2 s \varphi} d x d t \leq C \int_{Q(\varepsilon)} v^{2} e^{2 s \varphi} d x d t, \quad s \geq s_{0} .
$$

Similarly, from (2.7) we obtain

$$
\int_{Q(\varepsilon)}\left|\nabla_{x, t} u\right|^{2} e^{2 s \varphi} d x d t \leq C \int_{Q(\varepsilon)}\left(\left|\nabla_{x, t} v\right|^{2}+v^{2}\right) e^{2 s \varphi} d x d t, \quad s \geq s_{0} .
$$

Hence, substituting (2.8) and (2.9) into the left hand side of (2.6) and applying Lemma 1 to the third term on the right hand side of (2.6), we obtain

$$
\begin{aligned}
\int_{Q(\varepsilon)} & \left(s\left|\nabla_{x, t} u\right|^{2}+s^{3} u^{2}\right) e^{2 s \varphi} d x d t \\
\leq & C \int_{Q(\varepsilon)}\left(s\left|\nabla_{x, t} v\right|^{2}+\left(s+s^{3}\right) v^{2}\right) e^{2 s \varphi} d x d t \\
\leq & C \int_{Q(\varepsilon)}\left(s\left|\nabla_{x, t} v\right|^{2}+s^{3} v^{2}\right) e^{2 s \varphi} d x d t \\
\leq & C \int_{Q(\varepsilon)}\left(\left|\nabla_{x, t} u\right|^{2}+u^{2}\right) e^{2 s \varphi} d x d t \\
& +C \int_{Q(\varepsilon)} F^{2} e^{2 s \varphi} d x d t+C e^{C s}\|v\|_{(1), \Sigma}^{2} \\
\leq & C \int_{Q(\varepsilon)}\left(\left|\nabla_{x, t} u\right|^{2}+u^{2}\right) e^{2 s \varphi} d x d t \\
& +C \int_{Q(\varepsilon)} F^{2} e^{2 s \varphi} d x d t+C e^{C s}\|u\|_{(1), \Sigma}^{2}
\end{aligned}
$$

In order to derive the last inequality, we used

$$
\|v\|_{(1), \Sigma}^{2} \leq C\|u\|_{(1), \Sigma}^{2}
$$

by (2.1). Taking again $s>0$ sufficiently large, we absorb the first term on the right hand side into the left hand side at (2.10). Thus the proof of Theorem 1 is complete. 


\section{Proof of Theorem 2}

The proof is based on the modification by Imanuvilov and Yamamoto [10] of the original method by Bukhgeim and Klibanov [3]. The main ideas of the proof are as follows:

(1) In order to apply the Carleman estimate, the functions under consideration have to vanish on a part of $\partial(\Omega \times(0, T))$ (see (1.5)). Therefore we introduce a cut-off function given by (3.2).

(2) After taking the $t$-derivative of $u$, an unknown function $f=f(x)$ appears in the initial value and the right hand side $J$ (see (3.11)).

(3) Applying the Carleman estimate with large parameter $s>0$ to the $t$ differentiated equation, we can estimate the $L^{2}$-norm of $f(x)$ with the weight $e^{2 s \varphi(x, 0)}$ by $|J|$ and suitable norms of the boundary data on $\Gamma \times$ $(0, T)$ (see (3.16)-(3.17)).

(4) Thanks to the Carleman weight function, the coefficient of $|f(x)|^{2}$ in $J$ tends to 0 as $s \longrightarrow \infty$. Thus the term of $f$ in $J$ can be absorbed, so that the proof is complete.

Although our proof originates from [3], the steps (3)-(4) are different and are more convenient for deriving an estimate which is global over the whole domain $\Omega$.

We can prove now Theorem 2. First we modify Theorem 1 as follows.

Corollary 1. Let $u \in H^{2}(Q(\varepsilon))$ satisfy (1.1) and $u(x, 0)=0, x \in \Omega(\varepsilon)$. Then there exist $s_{0}>0$ and a constant $C=C\left(s_{0}\right)>0$ independent of $u$ such that

$$
\begin{aligned}
& \int_{Q(\varepsilon)}\left(s\left|\nabla_{x, t} u\right|^{2}+s^{3} u^{2}\right) e^{2 s \varphi} d x d t \leq C \int_{Q(\varepsilon)}|F|^{2} e^{2 s \varphi} d x d t \\
&+C e^{C s} \int_{\partial Q(\varepsilon) \cap(\Gamma \times(0, \infty))}\left(\left|\nabla_{x, t} u\right|^{2}+u^{2}\right) d S \\
&+C s^{3} e^{2 s\left(R^{2}+3 \varepsilon\right)}\|u\|_{H^{1}(Q(\varepsilon))}^{2}
\end{aligned}
$$

for any $s \geq s_{0}$. 
Proof of Corollary 1. Let $\chi \in C_{0}^{\infty}\left(\mathbb{R}^{n+1}\right)$ satisfy $0 \leq \chi \leq 1$ in $\mathbb{R}^{n+1}$ and

$$
\chi(x, t)= \begin{cases}1, & (x, t) \in Q(3 \varepsilon), \\ 0, & (x, t) \in Q(\varepsilon) \backslash Q(2 \varepsilon) .\end{cases}
$$

We set $v=\chi u$. Then $|v|=\left|\nabla_{x, t} v\right|=0$ on $\partial Q(\varepsilon) \backslash\{(\Gamma \times(0, \infty)) \cup(\Omega(\varepsilon)$ $\times\{0\})\}$ and $v=0$ on $\Omega(\varepsilon)$. Therefore Theorem 1 yields

$$
\begin{gathered}
\int_{Q(\varepsilon)}\left(s\left|\nabla_{x, t}(\chi u)\right|^{2}+s^{3}|\chi u|^{2}\right) e^{2 s \varphi} d x d t \leq C \int_{Q(\varepsilon)}|F|^{2} e^{2 s \varphi} d x d t \\
+C e^{C s} \int_{\partial Q(\varepsilon) \cap(\Gamma \times(0, \infty))}\left(\left|\nabla_{x, t}(\chi u)\right|^{2}+|\chi u|^{2}\right) d S
\end{gathered}
$$

for any $s \geq s_{0}$. Since

$$
\begin{gathered}
\int_{Q(\varepsilon)}\left(s\left|\nabla_{x, t} u\right|^{2}+s^{3} u^{2}\right) e^{2 s \varphi} d x d t \\
=\left(\int_{Q(3 \varepsilon)}+\int_{Q(\varepsilon) \backslash Q(3 \varepsilon)}\right)\left(s\left|\nabla_{x, t} u\right|^{2}+s^{3} u^{2}\right) e^{2 s \varphi} d x d t
\end{gathered}
$$

and $\chi=1$ in $Q(3 \varepsilon), \varphi(x, t) \leq R^{2}+3 \varepsilon$ for $(x, t) \in Q(\varepsilon) \backslash Q(3 \varepsilon)$, we have

$$
\begin{gathered}
\int_{Q(\varepsilon)}\left(s\left|\nabla_{x, t} u\right|^{2}+s^{3} u^{2}\right) e^{2 s \varphi} d x d t \\
\leq \int_{Q(3 \varepsilon)}\left(s\left|\nabla_{x, t}(\chi u)\right|^{2}+s^{3}|\chi u|^{2}\right) e^{2 s \varphi} d x d t+C s^{3} e^{2 s\left(R^{2}+3 \varepsilon\right)}\|u\|_{H^{1}(Q(\varepsilon))}^{2} .
\end{gathered}
$$

Thus the proof of Corollary 1 follows from this inequality and (3.3).

Now we proceed to proving Theorem 2. By (1.12), we have $\beta T^{2}>\left|x-x_{0}\right|^{2}$ for $x \in \Omega(0)$. Since $(x, t) \in Q(\varepsilon)$ implies that $x \in \Omega(0)$ and $\left|x-x_{0}\right|^{2}-\beta t^{2}>0$, we have $0<t<T$. Hence $Q(\varepsilon) \subset \Omega \times(0, T)$.

Let $u$ satisfy (1.8) and (1.9). For the sake of simplicity, we will make use of the shorthands:

$$
\left\{\begin{array}{l}
D=\|u\|_{H^{1}(\Gamma \times(0, T))}^{2}+\left\|\partial_{t} u\right\|_{H^{1}(\Gamma \times(0, T))}^{2}, \\
M=\|u\|_{H^{1}(Q(0))}^{2}+\left\|\partial_{t} u\right\|_{H^{1}(Q(0))}^{2}+\|f\|_{L^{2}(\Omega(0))}^{2},
\end{array}\right.
$$

where $D$ is a quantity depending only on the data, while $M$ is related to the $a$ priori bound of $u$ and $f$, needed to obtain the stability result (see (3.5) and (3.9)). 
Applying Corollary 1 to (1.8), we obtain

$$
\begin{gathered}
\int_{Q(\varepsilon)}\left(s\left|\nabla_{x, t} u\right|^{2}+s^{3} u^{2}\right) e^{2 s \varphi} d x d t \\
\leq C \int_{Q(\varepsilon)}|f|^{2} e^{2 s \varphi} d x d t+C e^{C s} D+C s^{3} e^{2 s\left(R^{2}+3 \varepsilon\right)} M, \quad s \geq s_{0} .
\end{gathered}
$$

On the other hand, (1.8) yields

$$
\begin{aligned}
\Delta u(x, t)= & -\int_{0}^{t} \frac{K(x, t, \eta)}{p(x)} \Delta u(x, \eta) d \eta+\frac{1}{p(x)} \partial_{t}^{2} u(x, t) \\
& -\frac{1}{p(x)} L(u)(x, t)-\frac{1}{p(x)} r(x, t) f(x), \quad(x, t) \in Q(\varepsilon) .
\end{aligned}
$$

Therefore Lemma 1 implies

$$
\begin{gathered}
\int_{Q(\varepsilon)}|\Delta u|^{2} e^{2 s \varphi} d x d t \leq \frac{C}{s} \int_{Q(\varepsilon)}|\Delta u|^{2} e^{2 s \varphi} d x d t+C \int_{Q(\varepsilon)}\left|\partial_{t}^{2} u\right|^{2} e^{2 s \varphi} d x d t \\
+C \int_{Q(\varepsilon)}\left(\left|\nabla_{x, t} u\right|^{2}+|u|^{2}\right) e^{2 s \varphi} d x d t+C \int_{Q(\varepsilon)} f^{2} e^{2 s \varphi} d x d t .
\end{gathered}
$$

Hence, for $s>0$ sufficiently large, we obtain

$$
\begin{gathered}
\int_{Q(\varepsilon)}|\Delta u|^{2} e^{2 s \varphi} d x d t \leq C \int_{Q(\varepsilon)}\left|\partial_{t}^{2} u\right|^{2} e^{2 s \varphi} d x d t \\
+C \int_{Q(\varepsilon)}\left(\left|\nabla_{x, t} u\right|^{2}+|u|^{2}\right) e^{2 s \varphi} d x d t+C \int_{Q(\varepsilon)} f^{2} e^{2 s \varphi} d x d t, \quad s \geq s_{0} .
\end{gathered}
$$

Next, setting $w=\partial_{t} u$, by (1.1) with $F=r f$ and (1.9) we have

$$
\begin{gathered}
\partial_{t}^{2} w(x, t)-p(x) \Delta w(x, t)=K(x, t, t) \Delta u(x, t) \\
+\int_{0}^{t}\left(\partial_{t} K\right)(x, t, \eta) \Delta u(x, \eta) d \eta+\partial_{t} L(u)(x, t)+\left(\partial_{t} r\right)(x, t) f(x), \\
(x, t) \in Q(\varepsilon)
\end{gathered}
$$

and $w(x, 0)=0$ for $x \in \Omega(\varepsilon)$. 
Noting that

$$
\begin{aligned}
\partial_{t} L(u)(x, t)= & \sum_{j=1}^{n} q_{j}(x) \partial_{j} \partial_{t} u(x, t)+q_{n+1}(x) \partial_{t}^{2} u(x, t) \\
& +q_{0}(x) \partial_{t} u(x, t)+H_{n+1}(x, t, t) \partial_{t} u(x, t) \\
& +\sum_{j=1}^{n} H_{j}(x, t, t) \partial_{j} u(x, t)+H_{0}(x, t, t) u(x, t) \\
& +\sum_{j=1}^{n} \int_{0}^{t} \partial_{t} H_{j}(x, t, \eta) \partial_{j} u(x, \eta) d \eta \\
& +\int_{0}^{t} \partial_{t} H_{n+1}(x, t, \eta) \partial_{t} u(x, \eta) d \eta \\
& +\int_{0}^{t} \partial_{t} H_{0}(x, t, \eta) u(x, \eta) d \eta, \quad x \in \Omega, 0<t<T,
\end{aligned}
$$

we have

$$
\begin{aligned}
& \left|\partial_{t} L(u)(x, t)\right| \leq C\left(\left|\nabla_{x, t} \partial_{t} u(x, t)\right|+\left|\nabla_{x, t} u(x, t)\right|+|u(x, t)|\right) \\
& +C \int_{0}^{t}\left(\left|\nabla_{x, t} u(x, \eta)\right|+|u(x, \eta)|\right) d \eta, \quad x \in \Omega, 0<t<T .
\end{aligned}
$$

Applying Corollary 1 to the function $w=\partial_{t} u$ and to the operator $\partial_{t}^{2}-p(x) \Delta$, corresponding to (1.1) with $L=K=0$, where $F$ is the right-hand side of the previous equation, we have

$$
\begin{gathered}
\int_{Q(\varepsilon)}\left(s\left|\nabla_{x, t} \partial_{t} u\right|^{2}+s^{3}\left|\partial_{t} u\right|^{2}\right) e^{2 s \varphi} d x d t \leq C \int_{Q(\varepsilon)}|f|^{2} e^{2 s \varphi} d x d t \\
\quad+C \int_{Q(\varepsilon)}\left(|\Delta u|^{2}+\left|\nabla_{x, t} \partial_{t} u\right|^{2}+\left|\nabla_{x, t} u\right|^{2}+|u|^{2}\right) e^{2 s \varphi} d x d t \\
\quad+C e^{C s} D+C s^{3} e^{2 s\left(R^{2}+3 \varepsilon\right)} M, \quad s \geq s_{0} .
\end{gathered}
$$

Hence, for large $s>0$, we deduce

$$
\begin{aligned}
\int_{Q(\varepsilon)} & \left(s\left|\nabla_{x, t} \partial_{t} u\right|^{2}+s^{3}\left|\partial_{t} u\right|^{2}\right) e^{2 s \varphi} d x d t \leq C \int_{Q(\varepsilon)}|f|^{2} e^{2 s \varphi} d x d t \\
& +C \int_{Q(\varepsilon)}\left(|\Delta u|^{2}+|\nabla u|^{2}+|u|^{2}\right) e^{2 s \varphi} d x d t \\
& +C e^{C s} D+C s^{3} e^{2 s\left(R^{2}+3 \varepsilon\right)} M .
\end{aligned}
$$


Combining (3.5), (3.6) and (3.9) and taking $s>0$ sufficiently large, we obtain

$$
\begin{aligned}
& \int_{Q(\varepsilon)}\left(|\Delta u|^{2}+s\left|\nabla_{x, t} u\right|^{2}+s\left|\nabla_{x, t} \partial_{t} u\right|^{2}+s^{3}\left|\partial_{t} u\right|^{2}+s^{3} u^{2}\right) e^{2 s \varphi} d x d t \\
& \quad \leq C \int_{Q(\varepsilon)}|f|^{2} e^{2 s \varphi} d x d t+C e^{C s} D+C s^{3} e^{2 s\left(R^{2}+3 \varepsilon\right)} M, \quad s \geq s_{0} .
\end{aligned}
$$

We now set $z=\chi\left(\partial_{t} u\right) e^{s \varphi}$. The introduction of the new function $z$ is convenient for estimating the initial value containing $f$ with the weight function $e^{2 s \varphi}$. Then we compute $\partial_{t}^{2} z$ and $\Delta z$ :

$$
\begin{aligned}
\partial_{t}^{2} z= & e^{s \varphi} \chi \partial_{t}^{3} u+e^{s \varphi}\left(\partial_{t}^{2} \chi\right) \partial_{t} u+s e^{s \varphi} \chi\left(\partial_{t} u\right)\left[\partial_{t}^{2} \varphi+s\left(\partial_{t} \varphi\right)^{2}\right] \\
& +2 e^{s \varphi}\left(\partial_{t} \chi\right) \partial_{t}^{2} u+2 s e^{s \varphi}\left(\partial_{t} \chi\right)\left(\partial_{t} u\right) \partial_{t} \varphi+2 s e^{s \varphi} \chi\left(\partial_{t}^{2} u\right) \partial_{t} \varphi, \\
\Delta z= & e^{s \varphi} \chi \Delta \partial_{t} u+e^{s \varphi}(\Delta \chi) \partial_{t} u+s e^{s \varphi} \chi \partial_{t} u\left[\Delta \varphi+s|\nabla \varphi|^{2}\right] \\
& +2 e^{s \varphi} \nabla \chi \cdot \nabla \partial_{t} u+2 s e^{s \varphi} \chi \nabla\left(\partial_{t} u\right) \cdot \nabla \varphi+2 s e^{s \varphi}\left(\partial_{t} u\right) \nabla \chi \cdot \nabla \varphi .
\end{aligned}
$$

By these formulae and (3.7), we deduce that $z$ solves the equation

$$
\begin{gathered}
\partial_{t}^{2} z-p \Delta z=\left[\chi\left\{\partial_{t} L(u)+\left(\partial_{t} r\right) f\right\}+\left(\partial_{t}^{2} \chi\right) \partial_{t} u\right. \\
+s \chi \partial_{t} u\left\{\partial_{t}^{2} \varphi+s\left(\partial_{t} \varphi\right)^{2}\right\}+2\left(\partial_{t} \chi\right) \partial_{t}^{2} u+2 s\left(\partial_{t} \chi\right)\left(\partial_{t} u\right) \partial_{t} \varphi \\
+2 s \chi\left(\partial_{t}^{2} u\right) \partial_{t} \varphi-p(x)(\Delta \chi) \partial_{t} u-s p(x) \chi \partial_{t} u\left\{\Delta \varphi+s|\nabla \varphi|^{2}\right\} \\
\left.-2 p(x) \nabla \chi \cdot \nabla \partial_{t} u-2 p(x) s \chi \nabla\left(\partial_{t} u\right) \cdot \nabla \varphi-2 s p(x)\left(\partial_{t} u\right) \nabla \chi \cdot \nabla \varphi\right] e^{s \varphi} \\
+\chi e^{s \varphi}\left\{K(x, t, t) \Delta u(x, t)+\int_{0}^{t}\left(\partial_{t} K\right)(x, t, \eta) \Delta u(x, \eta) d \eta\right\} \equiv J(u) .
\end{gathered}
$$

Then we have

$$
\begin{gathered}
|J(u)(x, t)| \leq C e^{s \varphi}\left(s\left|\nabla_{x, t} u(x, t)\right|+|u(x, t)|\right. \\
\left.+s\left|\nabla_{x, t}\left(\partial_{t} u\right)(x, t)\right|+s^{2}\left|\partial_{t} u(x, t)\right|+|\Delta u(x, t)|\right) \\
+C e^{s \varphi}|f(x)|+C e^{s \varphi} \int_{0}^{t}\left(\left|\nabla_{x, t} u(x, \eta)\right|+|u(x, \eta)|+|\Delta u(x, \eta)|\right) d \eta, \\
(x, t) \in Q(\varepsilon) .
\end{gathered}
$$

Multiply

$$
-\partial_{t}^{2} z+p \Delta z=-J(u) \quad \text { by } \quad 2 \partial_{t} z
$$


and integrate over $Q(\varepsilon)$ to obtain

$$
\begin{gathered}
-\int_{Q(\varepsilon)} 2\left(\partial_{t}^{2} z\right) \partial_{t} z d x d t+\int_{Q(\varepsilon)} 2\left(\partial_{t} z\right) p \Delta z d x d t \\
=-2 \int_{Q(\varepsilon)} J(u)\left(\partial_{t} z\right) d x d t .
\end{gathered}
$$

We see that

$$
\left|\partial_{t} z(x, t)\right| \leq C s\left|\partial_{t} u(x, t)\right| e^{s \varphi}+C\left|\partial_{t}^{2} u(x, t)\right| e^{s \varphi}, \quad(x, t) \in Q(\varepsilon)
$$

and

$$
|\nabla z(x, t)| \leq C s\left|\partial_{t} u(x, t)\right| e^{s \varphi}+C\left|\nabla_{x, t} \partial_{t} u(x, t)\right| e^{s \varphi}, \quad(x, t) \in Q(\varepsilon) .
$$

Henceforth let $\left(v, v_{n+1}\right)=\left(v_{1}, \ldots, v_{n}, v_{n+1}\right)$ denote the unit outward normal vector to $\partial Q(\varepsilon)$. Hence, in terms of (1.9) and (3.2), we obtain that $z=$ $\left|\nabla_{x, t} z\right|=0$ on $\partial Q(\varepsilon) \backslash(\Gamma \times(0, T)) \backslash(\Omega(\varepsilon) \times\{0\}), \nabla z=0$ on $\Omega(\varepsilon) \times\{0\}$ and $v_{n+1}=0$ on $\partial Q(\varepsilon) \cap(\Gamma \times(0, T))$. An integration by parts gives

$$
\begin{aligned}
- & \int_{Q(\varepsilon)} 2\left(\partial_{t}^{2} z\right) \partial_{t} z d x d t+\int_{Q(\varepsilon)} 2\left(\partial_{t} z\right) p \Delta z d x d t \\
= & -\int_{Q(\varepsilon)} \partial_{t}\left(\left|\partial_{t} z\right|^{2}\right) d x d t-\int_{Q(\varepsilon)} p \partial_{t}\left(|\nabla z|^{2}\right) d x d t \\
& +\int_{\partial Q(\varepsilon)} 2\left(\partial_{t} z\right) p \nabla z \cdot v d S-2 \int_{Q(\varepsilon)} \nabla p \cdot(\nabla z)\left(\partial_{t} z\right) d x d t \\
= & \int_{\Omega(\varepsilon)}\left|\partial_{t} z(\cdot, 0)\right|^{2} d x+2 \int_{\partial Q(\varepsilon) \cap(\Gamma \times(0, T))} p\left(\partial_{t} z\right) \nabla z \cdot v d S \\
& -2 \int_{Q(\varepsilon)} \nabla p \cdot(\nabla z)\left(\partial_{t} z\right) d x d t \quad p\left(\partial_{t} z\right) \nabla z \cdot v d S \\
\geq & \int_{\Omega(\varepsilon)}\left|\partial_{t} z(\cdot, 0)\right|^{2} d x+2 \int_{\partial Q(\varepsilon) \cap(\Gamma \times(0, T))} \\
& -C \int_{Q(\varepsilon)}\left(|z|^{2}+\left|\partial_{t} z\right|^{2}\right) d x d t .
\end{aligned}
$$


Hence

$$
\begin{gathered}
\int_{\Omega(\varepsilon)}\left|\partial_{t} z(\cdot, 0)\right|^{2} d x \\
\leq-2 \int_{Q(\varepsilon)} J(u)\left(\partial_{t} z\right) d x d t+2 \int_{\partial Q(\varepsilon) \cap(\Gamma \times(0, T))}|p|\left|\partial_{t} z\right||\nabla z \cdot v| d S \\
+\int_{Q(\varepsilon)}\left(s^{2}\left|\partial_{t} u\right|^{2}+\left|\nabla_{x, t} \partial_{t} u\right|^{2}\right) e^{2 s \varphi} d x d t .
\end{gathered}
$$

By (3.12) we have

$$
\begin{gathered}
\left|-2 \int_{Q(\varepsilon)} J(u) \partial_{t} z d x d t\right| \\
\leq C \int_{Q(\varepsilon)}\left(s\left|\nabla_{x, t} u\right|+|u|+s\left|\nabla_{x, t} \partial_{t} u\right|+s^{2}\left|\partial_{t} u\right|+|\Delta u|\right)\left(\left|\partial_{t}^{2} u\right|+s\left|\partial_{t} u\right|\right) e^{2 s \varphi} d x d t \\
+C \int_{Q(\varepsilon)}|f|\left(\left|\partial_{t}^{2} u\right|+s\left|\partial_{t} u\right|\right) e^{2 s \varphi} d x d t \\
+C \int_{Q(\varepsilon)} e^{2 s \varphi}\left(\left|\partial_{t}^{2} u\right|+s\left|\partial_{t} u\right|\right)\left(\int_{0}^{t}\left(\left|\nabla_{x, t} u(x, \eta)\right|+|u(x, \eta)|+|\Delta u(x, \eta)|\right) d \eta\right) d x d t .
\end{gathered}
$$

On the other hand, the Cauchy-Schwarz inequality yields

$$
s^{2}\left|\nabla_{x, t} \partial_{t} u\right|\left|\partial_{t} u\right| \leq s\left|\nabla_{x, t} \partial_{t} u\right|^{2}+s^{3}\left|\partial_{t} u\right|^{2}
$$

and

$$
|f|\left(\left|\partial_{t}^{2} u\right|+s\left|\partial_{t} u\right|\right) \leq|f|^{2}+2\left|\partial_{t}^{2} u\right|^{2}+2 s^{2}\left|\partial_{t} u\right|^{2},
$$

etc. Taking advantage of Lemma 1 , we derive the estimate

$$
\begin{gathered}
\left|-2 \int_{Q(\varepsilon)} J(u) \partial_{t} z d x d t\right| \\
\leq C \int_{Q(\varepsilon)}\left(|u|^{2}+|\Delta u|^{2}+s\left|\nabla_{x, t} u\right|^{2}+s\left|\nabla_{x, t} \partial_{t} u\right|^{2}+s^{3}\left|\partial_{t} u\right|^{2}\right) e^{2 s \varphi} d x d t \\
+C \int_{Q(\varepsilon)}|f|^{2} e^{2 s \varphi} d x d t .
\end{gathered}
$$

Hence inequality (3.10) yields

$$
\begin{gathered}
\left|-2 \int_{Q(\varepsilon)} J\left(\partial_{t} z\right) d x d t\right| \\
\leq C \int_{Q(\varepsilon)} f^{2} e^{2 s \varphi} d x d t+C e^{C s} D+C s^{3} e^{2 s\left(R^{2}+3 \varepsilon\right)} M, \quad s \geq s_{0} .
\end{gathered}
$$


Consequently, recalling definition (3.4) of $D$, from (3.13)-(3.15), we derive

$$
\begin{gathered}
\int_{\Omega(\varepsilon)}\left|\partial_{t} z(x, 0)\right|^{2} d x \leq C \int_{\Gamma \times(0, T)}\left(\left|\partial_{t} z\right|^{2}+|\nabla z|^{2}\right) d S \\
+C \int_{Q(\varepsilon)} f^{2} e^{2 s \varphi} d x d t+C e^{C s} D+C s^{3} e^{2 s\left(R^{2}+3 \varepsilon\right)} M \\
\leq C \int_{Q(\varepsilon)} f^{2} e^{2 s \varphi} d x d t+C e^{C s} D+C s^{3} e^{2 s\left(R^{2}+3 \varepsilon\right)} M, \quad s \geq s_{0} .
\end{gathered}
$$

By (1.8) and (1.9), we have

$$
\left(\partial_{t} z\right)(x, 0)=\chi(x, 0)\left(\partial_{t}^{2} u\right)(x, 0) e^{s \varphi(x, 0)}=\chi(x, 0) r(x, 0) f(x) e^{s \varphi(x, 0)}
$$

for $x \in \Omega(\varepsilon)$. Hence, (1.11), (3.2) and (3.16) imply

$$
\begin{gathered}
\int_{\Omega(3 \varepsilon)} f^{2} e^{2 s \varphi(x, 0)} d x \leq C \int_{\Omega(\varepsilon)}\left|\partial_{t} z(x, 0)\right|^{2} d x \\
\leq C \int_{Q(\varepsilon)} f^{2} e^{2 s \varphi} d x d t+C e^{C s} D+C s^{3} e^{2 s\left(R^{2}+3 \varepsilon\right)} M, \quad s \geq s_{0} .
\end{gathered}
$$

Consider now the inequalities

$$
\begin{gathered}
\int_{Q(3 \varepsilon)} f^{2} e^{2 s \varphi} d x d t \\
=\int_{\Omega(3 \varepsilon)}|f(x)|^{2} e^{2 s \varphi(x, 0)}\left(\int_{0}^{\left(\left|x-x_{0}\right|^{2}-\left(R^{2}+3 \varepsilon\right)\right)^{\frac{1}{2}} \beta^{-\frac{1}{2}}} e^{2 s(\varphi(x, t)-\varphi(x, 0))} d t\right) d x \\
\leq \int_{\Omega(3 \varepsilon)}|f(x)|^{2} e^{2 s \varphi(x, 0)}\left(\int_{0}^{+\infty} e^{-2 s \beta t^{2}} d t\right) d x \\
=\frac{\sqrt{\pi}}{2 \sqrt{2 \beta}} \frac{1}{\sqrt{s}} \int_{\Omega(3 \varepsilon)}|f(x)|^{2} e^{2 s \varphi(x, 0)} d x
\end{gathered}
$$

and

$$
\int_{Q(\varepsilon) \backslash Q(3 \varepsilon)} f^{2} e^{2 s \varphi} d x d t \leq C M e^{2 s\left(R^{2}+3 \varepsilon\right)}
$$

Hence

$$
\begin{gathered}
\int_{Q(\varepsilon)}|f(x)|^{2} e^{2 s \varphi} d x d t=\left(\int_{Q(3 \varepsilon)}+\int_{Q(\varepsilon) \backslash Q(3 \varepsilon)}\right)|f(x)|^{2} e^{2 s \varphi} d x d t \\
\leq \frac{C}{\sqrt{s}} \int_{\Omega(3 \varepsilon)}|f(x)|^{2} e^{2 \varphi(x, 0)} d x+C M e^{2 s\left(R^{2}+3 \varepsilon\right)}
\end{gathered}
$$


Therefore from (3.17), we deduce

$$
\begin{gathered}
\int_{\Omega(3 \varepsilon)} f^{2} e^{2 s \varphi(x, 0)} d x \\
\leq \frac{C}{\sqrt{s}} \int_{\Omega(3 \varepsilon)} f^{2} e^{2 s \varphi(x, 0)} d x+C e^{C s} D+C s^{3} e^{2 s\left(R^{2}+3 \varepsilon\right)} M, \quad s \geq s_{0} .
\end{gathered}
$$

Hence, for sufficiently large $s$, we obtain

$$
\int_{\Omega(3 \varepsilon)} f^{2} e^{2 s \varphi(x, 0)} d x \leq C e^{C s} D+C s^{3} e^{2 s\left(R^{2}+3 \varepsilon\right)} M, \quad s \geq s_{0} .
$$

Consequently

$$
\begin{gathered}
e^{2 s\left(R^{2}+4 \varepsilon\right)}\|f\|_{L^{2}(\Omega(4 \varepsilon))}^{2} \leq \int_{\Omega(4 \varepsilon)}|f(x)|^{2} e^{2 s \varphi(x, 0)} d x \\
\leq \int_{\Omega(3 \varepsilon)}|f(x)|^{2} e^{2 s \varphi(x, 0)} d x \leq C e^{C s} D+C s^{3} e^{2 s\left(R^{2}+3 \varepsilon\right)} M, \quad s \geq s_{0},
\end{gathered}
$$

that is,

$$
\|f\|_{L^{2}(\Omega(4 \varepsilon))}^{2} \leq C e^{C s} D+C s^{3} e^{-2 \varepsilon s} M \leq C e^{C s} D+C e^{-\varepsilon s} M, \quad s \geq s_{0}
$$

for a suitable $C>0$. Then we replace $C>0$ with $C e^{C s_{0}}$ so that (3.18) holds for all $s>0$. Assume $M>D$ and choose $s=\frac{1}{C+\varepsilon} \log \frac{M}{D}>0$. Then we obtain

$$
\|f\|_{L^{2}(\Omega(4 \varepsilon))}^{2} \leq 2 C M^{\frac{C}{C+\varepsilon}} D^{\frac{\varepsilon}{C+\varepsilon}} .
$$

If $M \leq D$, then the proof is already complete. Choosing $\delta=4 \varepsilon$, we conclude the proof of Theorem 2 .

\section{Appendix. Proof of Lemma 1.}

First we have

$$
t e^{2 s \varphi(x, t)}=-\frac{1}{4 \beta s} \partial_{t}\left(e^{2 s \varphi}\right) .
$$

Therefore, by the Cauchy-Schwarz inequality, we obtain

$$
\begin{gathered}
\int_{Q(\varepsilon)}\left(\int_{0}^{t}|w(x, \xi)| d \xi\right)^{2} e^{2 s \varphi} d x d t \leq \int_{Q(\varepsilon)} t\left(\int_{0}^{t}|w(x, \xi)|^{2} d \xi\right) e^{2 s \varphi} d x d t \\
\leq \int_{\Omega(\varepsilon)}\left\{\int_{0}^{\ell(x)}-\frac{1}{4 \beta s} \partial_{t}\left(e^{2 s \varphi}\right)\left(\int_{0}^{t}|w(x, \xi)|^{2} d \xi\right) d t\right\} d x
\end{gathered}
$$


Here we have set $\ell(x)=\left(\frac{\left[\left|x-x_{0}\right|^{2}-R^{2}-\varepsilon\right]}{\beta}\right)^{1 / 2}$. An integration by parts yields

$$
\begin{gathered}
\int_{Q(\varepsilon)}\left(\int_{0}^{t}|w(x, \xi)| d \xi\right)^{2} e^{2 s \varphi} d x d t \\
\leq \frac{1}{4 \beta s}\left\{-e^{2 s\left(R^{2}+\varepsilon\right)} \int_{\Omega(\varepsilon)}\left(\int_{0}^{\ell(x)}|w(x, \xi)|^{2} d \xi\right) d x+\int_{Q(\varepsilon)}|w(x, \xi)|^{2} e^{2 s \varphi} d x d t\right\} \\
\leq \frac{1}{4 \beta s} \int_{Q(\varepsilon)}|w(x, \xi)|^{2} e^{2 s \varphi} d x d t .
\end{gathered}
$$

The proof of Lemma 1 is complete.

Acknowledgements. The first and the second authors are members of the G.N.A.M.P.A. of the Italian C.N.R. and were partially supported by by the Italian Ministero dell'Università e della Ricerca Scientifica e Tecnologica, PRIN no. 2004011204, project Analisi Matematica nei Problemi Inversi. The third named author was supported partially by Grant 15340027 from the Japan Society for the Promotion of Science and Grant 17654019 from the Ministry of Education, Cultures, Sports and Technology. The authors thank the anonymous referee for his/her careful reading and valuable comments.

\section{REFERENCES}

[1] M. Bellassoued, Global logarithmic stability in inverse hyperbolic problem by arbitrary boundary observation. Inverse Problems, 20 (2004), 1033-1052.

[2] A.L. Bukhgeim, Introduction to the Theory of Inverse Problems. VSP, Utrecht, 2000.

[3] A.L. Bukhgeim and M.V. Klibanov, Global uniqueness of class of multidimensional inverse problems. Soviet Math. Dokl., 24 (1981), 244-247.

[4] C. Cavaterra, An inverse problem for a viscoelastic Timoshenko beam model. Z. für Analysis und ihre Anwendungen 17 (1998), 67-87.

[5] C. Cavaterra and M. Grasselli, On an inverse problem for a model of linear viscoelastic Kirchhoff plate. J. Intergal Equations and Appl., 9 (1997), 179-218.

[6] C. Cavaterra and A. Lorenzi, An identification problem for the Maxwell equations in a non homogeneous dispersive medium. Diff. Int. Equations, 8 (1995), 167-190.

[7] J. Cheng, V. Isakov, M. Yamamoto and Q. Zhou, Lipschitz stability in the lateral Cauchy problem for elasticity system. J. Math. Kyoto Univ., 43 (2003), 475-501. 
[8] L. Hörmander, Linear Partial Differential Operators. Springer-Verlag, Berlin, 1963.

[9] O.Yu. Imanuvilov and M. Yamamoto, Global uniqueness and stability in determining coefficients of wave equations. Comm. Partial Differential Equations, 26 (2001), 1409-1425.

[10] O.Yu. Imanuvilov and M. Yamamoto, Global Lipschitz stability in an inverse hyperbolic problem by interior observations. Inverse Problems, 17 (2001), 717-728.

[11] O. Yu. Imanuvilov and M. Yamamoto, Carleman estimates for the non-stationary Lame system and the application to an inverse problem. ESAIM Control Optim. Calc. Var., 11 (2005), 1-56.

[12] V. Isakov, Inverse Source Problems. American Mathematical Society, Providence, Rhode Island, 1990.

[13] V. Isakov, Carleman type estimates in an anisotropic case and applications. J. Differential Equations, 105 (1993), 217-238.

[14] V. Isakov, Inverse Problems for Partial Differential Equations. Springer-Verlag, Berlin, 2005.

[15] J. Janno and A. Lorenzi, A parabolic integrodifferential identification problem in a barrelled smooth domain. ZAA, 25 (2006), 103-130.

[16] J. Janno and von Wolfersdorf, Inverse problems for identification of memory kernels in thermo- and poro-viscoelasticity. Math. Methods in Appl. Sci., 21 (1998), 1495-1517.

[17] S.I. Kabanikhin and A. Lorenzi, Identification Problems of Wave Phenomena. VSP, Utrecht, 1999.

[18] A. Khă̌darov, Carleman estimates and inverse problems for second order hyperbolic equations. Math. USSR Sbornik, 58 (1987), 267-277.

[19] M.V. Klibanov, Inverse problems and Carleman estimates. Inverse Problems, 8 (1992), $575-596$.

[20] M.V. Klibanov and A. Timonov, Carleman Estimates for Coefficient Inverse Problems and Numerical Applications. VSP, Utrecht, 2004.

[21] M.V. Klibanov and M. Yamamoto, Lipschitz stability of an inverse problem for an acoustic equation. Appl. Anal., 85 (2006), 515-538.

[22] M. Kubo, Uniqueness in inverse hyperbolic problems -Carleman estimate for boundary value problems. J. Math. Kyoto Univ., 40 (2000), 451-473.

[23] M.M.Lavrent'ev, V.G. Romanov and S.P. Shishat-skiŭ, Ill-posed Problems of Mathematical Physics and Analysis. American Mathematical Society, Providence, Rhode Island, 1986.

[24] A. Lorenzi, A multidimensional identification problem related to a hyperbolic integrodifferential equation. Z. für Analysis und ihre Anwendungen, 18 (1999), 407-435.

[25] A. Lorenzi and F. Messina, Identification problems for Maxwell integro-differential equations related to media with cylindric symmetries. J. Inverse and Ill-posed Problems, 11 (2003), 411-437. 
[26] A. Lorenzi and F. Messina, Gevrey type results in the identification of lower order coeffcients in linear hyperbolic integrodifferential equations. J. Inverse and Ill-posed Problems, 12 (2004), 1-40.

[27] A. Lorenzi and V. G. Romanov, Stability estimates for an inverse problem related to viscoelastic media, to appear in J. Inverse and Ill-Posed Problems (2006).

[28] A. Lorenzi and V.G. Yakhno, An identification problem related to an isotropic stratified viscoelastic cylindrical body. J. Inverse and Ill-Posed Problems (2005) 5 (1997), 29-53.

[29] L. von Wolfersdorf, On identification of memeory kernels in linear viscoelasticity. Math. Nachrichten, 161 (1993), 203-217.

[30] M. Yamamoto, Uniqueness and stability in multidimensional hyperbolic inverse problems. J. Math. Pures Appl., 78 (1999), 65-98.

[31] J. Yong and X. Zhang, Exact controllability of the heat equation with hyperbolic memory kernel, in "Control Theory of Partial Differential Equations", Chapman \& Hall/CRC, Boca Raton, (2005), 387-401. 